\title{
Harmonic Maps and Bi-Harmonic Maps on CR-Manifolds and Foliated Riemannian Manifolds
}

\author{
Shinji Ohno'1, Takashi Sakai², Hajime Urakawa ${ }^{3}$ \\ ${ }^{1}$ Osaka City University Advanced Mathematical Institute (OCAMI), Osaka, Japan \\ ${ }^{2}$ Department of Mathematics and Information Sciences, Tokyo Metropolitan University, Hachioji, Japan \\ ${ }^{3}$ Institute for International Education, Global Learning Center, Tohoku University, Sendai, Japan \\ Email: urakawa@math.is.tohoku.ac.jp
}

How to cite this paper: Ohno, S., Sakai, T. and Urakawa, H. (2016) Harmonic Maps and Bi-Harmonic Maps on CR-Manifolds and Foliated Riemannian Manifolds. Jour nal of Applied Mathematics and Physics, 4, 2272-2289.

http://dx.doi.org/10.4236/jamp.2016.412219

Received: July 19, 2016

Accepted: December 26, 2016

Published: December 29, 2016

Copyright $\odot 2016$ by authors and Scientific Research Publishing Inc. This work is licensed under the Creative Commons Attribution International License (CC BY 4.0).

http://creativecommons.org/licenses/by/4.0/

\begin{abstract}
This is a survey on our recent works on bi-harmonic maps on CR-manifolds and foliated Riemannian manifolds, and also a research paper on bi-harmonic maps principal $G$-bundles. We will show, (1) for a complete strictly pseudoconvex $C R$ manifold $\left(M, g_{\theta}\right)$, every pseudo bi-harmonic isometric immersion $\varphi:\left(M, g_{\theta}\right) \rightarrow(N, h)$ into a Riemannian manifold of non-positive curvature, with finite energy and finite bienergy, must be pseudo harmonic; (2) for a smooth foliated map of a complete, possibly non-compact, foliated Riemannian manifold into another foliated Riemannian manifold, of which transversal sectional curvature is non-positive, we will show that if it is transversally bi-harmonic map with the finite energy and finite bienergy, then it is transversally harmonic; (3) we will claim that the similar result holds for principal $G$-bundle over a Riemannian manifold of negative Ricci curvature.
\end{abstract}

\section{Keywords}

Foliation, Divergence Theorem, Transversally Harmonic, Transversally Biharmonic

\section{Introduction}

The theory of harmonic maps has been extensively developed and applied in many problems in topology and differential geometry (cf. [1] [2] [3], etc.). Eells and Lemaire raised ([3]) a problem to study $k$-harmonic maps and G. Y. Jiang calculated [4] the first variational and second formulas of the bienergy.

On the other hand, B.Y. Chen proposed [5] the famous conjecture in the study of sub-manifolds in the Euclidean space. B. Y. Chen's conjecture and the generalized B. Y. Chen's conjecture are as follows: 
The B. Y. Chen's conjecture: Every biharmonic isometric immersion into the Euclidean space $\mathbb{R}^{n}$ must be harmonic (minimal).

The generalized B. Y. Chen's conjecture: Every biharmonic isometric immersion of a Riemannian manifold $(M, g)$ into a Riemannian manifold $(N, h)$ of non-positive curvature must be harmonic (minimal).

The B. Y. Chen's conjecture is still open, but the generalized B. Y. Chen's conjecture was solved negatively by Ye-Lin Ou and Liang Tang [6], due to several authors have contributed to give partial answers to solve these problems (cf. [7]-[17]).

For the first and second variational formula of the bienergy, see [4].

Then, the $C R$ analogue for harmonic maps and biharmonic maps has been raised as follows.

The $C R$ analogue of the generalized Chen's conjecture: Let $\left(M, g_{\theta}\right)$ be a complete strictly pseudoconvex $C R$ manifold, and $(N, h)$, a Riemannian manifold of non-positive curvature. Then, every pseudo biharmonic isometric immersion $\varphi:\left(M, g_{\theta}\right) \rightarrow(N, h)$ must be pseudo harmonic.

For the works on $C R$ analogue of biharmonic maps, see [18] [19] [20]. We will show (cf. [20]):

Theorem 1.1. (cf. Theorem 2.1) Let $\varphi$ be a pseudo biharmonic map of a strictly pseudoconvex complete $C R$ manifold $\left(M, g_{\theta}\right)$ into another Riemannian manifold $(N, h)$ of non positive curvature.

If $\varphi$ has finite pseudo bienergy $E_{b, 2}(\varphi)<\infty$ and finite pseudo energy $E_{b}(\varphi)<\infty$, then it is pseudo harmonic, i.e., $\tau_{b}(\varphi)=0$.

Next, let us consider the analogue of harmonic maps and biharmonic maps for foliations are also given as follows. Transversally biharmonic maps between two foliated Riemannian manifolds were introduced by Chiang and Wolak (cf. [21]) and see also [22] [23] [24] [25] [26]. They are generalizations of transversally harmonic maps introduced by Konderak and Wolak (cf. [27] [28]).

Among smooth foliated maps $\varphi$ between two Riemannian foliated manifolds, one can define the transversal energy and derive the Euler-Lagrange equation, and transversally harmonic map as its critical points which are by definition the transversal tension field vanishes, $\tau_{b}(\varphi)=0$. The transverse bienergy can be also defined as $E_{2}(\varphi)=\frac{1}{2} \int_{M}\left|\tau_{b}(\varphi)\right|^{2} v_{g}$ whose Euler-Lagrange equation is that the transversal bitension field $\tau_{2, b}(\varphi)$ vanishes and the transversally biharmonic maps which are, by definition, vanishing of the transverse bitension field.

Recently, S.D. Jung studied extensively the transversally harmonic maps and the transversally biharmonic maps on compact Riemannian foliated manifolds (cf. [29] [30] [31] [32]).

Then, we will study transversally biharmonic maps of a complete (possibly noncompact) Riemannian foliated manifold $(M, g, \mathcal{F})$ into another Riemannian foliated manifold $\left(M^{\prime}, g^{\prime}, \mathcal{F}^{\prime}\right)$ of which transversal sectional curvature is non-positive. Then, we will show (cf. [33]) that: 
Theorem 1.2. (cf. Theorem 2.6) Let $(M, g, \mathcal{F})$ and $\left(M^{\prime}, g^{\prime}, \mathcal{F}^{\prime}\right)$ be two Riemannian foliated manifolds, and assume that the transversal sectional curvature of $\left(M^{\prime}, g^{\prime}, \mathcal{F}^{\prime}\right)$ is non-positive. Let $\varphi:(M, g, \mathcal{F}) \rightarrow\left(M^{\prime}, g^{\prime}, \mathcal{F}^{\prime}\right)$ be a smooth foliated map which is an isometric immersion of $(M, g)$ into $\left(M^{\prime}, g^{\prime}\right)$. If $\varphi$ is transversally biharmonic with the finite transversal energy $E(\varphi)<\infty$ and finite transversal bienergy $E_{2}(\varphi)<\infty$, then it is transversally harmonic.

Finally, in Section 5, instead of isometric immersions, we will consider a principal $G$-bundle $\pi:(P, g) \rightarrow(M, h)$, and show a new result whose details will be appeared in [34].

Theorem 1.3. (cf. Theorem 5.1) Let $\pi:(P, g) \rightarrow(M, h)$ be a principal G-bundle over a Riemannian manifold $(M, h)$ whose Ricci tensor is negative definite. Then, if $\pi:(P, g) \rightarrow(M, h)$ is biharmonic, then it is harmonic.

\section{Preliminaries}

\subsection{First and Second Variational Formulas for the Energy}

First, let us recall the theory of harmonic maps. For a smooth map $\varphi$ of a Riemannian manifold $(M, g)$ into another Riemannian manifold $(N, h)$, the energy functional $E(\bullet)$ is defined by

$$
E(\varphi):=\frac{1}{2} \int_{M}|\mathrm{~d} \varphi|^{2} v_{g}
$$

whose first variational formula is:

$$
\left.\frac{\mathrm{d}}{\mathrm{d} t}\right|_{t=0} E\left(\varphi_{t}\right)=-\int_{M}\langle\tau(\varphi), V\rangle v_{g}
$$

Here, $V$ is a variational vector field is given by $V_{x}=\left.\frac{\mathrm{d}}{\mathrm{d} t}\right|_{t=0} \varphi_{t}(x) \in T_{\varphi(x)} N, \quad(x \in M)$, and the tension field $\tau(\varphi)$ is given by

$$
\begin{aligned}
& \tau(\varphi):=\sum_{i=1}^{m} B(\varphi)\left(e_{i}, e_{i}\right), \\
& B(\varphi)(X, Y):=\nabla_{\mathrm{d} \varphi(X)}^{h} \mathrm{~d} \varphi(Y)-\mathrm{d} \varphi\left(\nabla_{X}^{g} Y\right), X, Y \in \mathfrak{X}(M),
\end{aligned}
$$

where $\nabla^{g}$ and $\nabla^{h}$ are Levi-Civita connections of $(M, g)$ and $(N, h)$, respectively. Then, $\varphi:(M, g) \rightarrow(N, h)$ is harmonic if $\tau(\varphi)=0$.

The second variation formula of the energy functional $E(\bullet)$ for a harmonic map $\varphi:(M, g) \rightarrow(N, h)$ is:

$$
\left.\frac{\mathrm{d}^{2}}{\mathrm{~d} t^{2}}\right|_{t=0} E\left(\varphi_{t}\right)=\int_{M}\langle J(V), V\rangle v_{g},
$$

where

$$
\begin{aligned}
& J(V):=\bar{\Delta} V-\mathcal{R}(V), \\
& \bar{\Delta} V:=\bar{\nabla}^{*} \bar{\nabla} V, \quad \mathcal{R}(V):=\sum_{i=1}^{m} R^{h}\left(V, \mathrm{~d} \varphi\left(e_{i}\right)\right) \mathrm{d} \varphi\left(e_{i}\right),
\end{aligned}
$$


where $\left\{e_{i}\right\}_{i=1}^{m}$ is a locally defined frame field on $(M, g)$. The $k$-energy functional due to J. Eells and L. Lemaire ([1] [2] [3]) is

$$
E_{k}(\varphi):=\frac{1}{2} \int_{M}\left|(d+\delta)^{k} \varphi\right|^{2} v_{g} \quad(k=1,2, \cdots)
$$

which turn out that

$$
E_{1}(\varphi)=\frac{1}{2} \int_{M}|\mathrm{~d} \varphi|^{2} v_{g}, \quad E_{2}(\varphi)=\frac{1}{2} \int_{M}|\tau(\varphi)|^{2} v_{g} .
$$

Furthermore, the first variation formula for $E_{2}(\varphi)$ is (cf. [4]):

$$
\begin{aligned}
& \left.\frac{\mathrm{d}}{\mathrm{d} t}\right|_{t=0} E_{2}\left(\varphi_{t}\right)=-\int_{M}\left\langle\tau_{2}(\varphi), V\right\rangle v_{g}, \\
& \tau_{2}(\varphi):=J(\tau(\varphi))=\bar{\nabla} \tau(\varphi)-\mathcal{R}(\tau(\varphi)) .
\end{aligned}
$$

Then, one can define that $\varphi:(M, g) \rightarrow(N, h)$ is biharmonic (cf. [4]) if $\tau_{2}(\varphi)=0$.

\subsection{The $C R$ Analogue of the Generalized Chen's Conjecture}

In this part, we first raise the $C R$ analogue of the generalized Chen's conjecture, and settle it for pseudo biharmonic maps with finite pseudo energy and finite pseudo bienergy.

Let us recall a strictly pseudoconvex $C R$ manifold (possibly non compact) $\left(M, g_{\theta}\right)$ of $(2 n+1)$-dimension, and the Webster Riemannian metric $g_{\theta}$ given by

$$
g_{\theta}(X, Y)=(\mathrm{d} \theta)(X, J Y), \quad g_{\theta}(X, T)=0, \quad g_{\theta}(T, T)=1
$$

for $X, Y \in H(M)$. Recall the material on the Levi-Civita connection $\nabla^{g_{\theta}}$ of $\left(M, g_{\theta}\right)$. Due to Lemma 1.3, Page 38 in [35], it holds that,

$$
\nabla^{g_{\theta}}=\nabla+(\Omega-A) \otimes T+\tau \otimes \theta+2 \theta \odot J,
$$

where $\nabla$ is the Tanaka-Webster connection, $\Omega=\mathrm{d} \theta$, and $A(X, Y)=g_{\theta}(\tau X, Y)$, $\tau X=T_{\nabla}(T, X)$, and $T_{\nabla}$ is the torsion tensor of $\nabla$. And also, $(\tau \otimes \theta)(X, Y)=\theta(Y) \tau X$, $(\theta \odot J)(X, Y)=\frac{1}{2}\{\theta(X) J Y+\theta(Y) J X\}$ for all vector fields $X, Y$ on $M$. Here, $J$ is the complex structure on $H(M)$ and is extended as an endomorphism on $(M)$ by $J T=0$.

Then, we have

$$
\begin{aligned}
& \nabla_{X_{k}}^{g_{\theta}} X_{k}=\nabla_{X_{k}} X_{k}-A\left(X_{k}, X_{k}\right) T, \\
& \nabla_{T}^{g_{\theta}} T=0,
\end{aligned}
$$

where $\left\{X_{k}\right\}_{k=1}^{2 n}$ is a locally defined orthonormal frame field on $H(M)$ with respect to $g_{\theta}$ and $T$ is the characteristic vector field of $\left(M, g_{\theta}\right)$. For (3.6), it follows from that $\Omega\left(X_{k}, X_{k}\right)=0,(\tau \otimes \theta)\left(X_{k}, X_{k}\right)=0$ and $(\theta \odot J)\left(X_{k}, X_{k}\right)=0$ since $\theta\left(X_{k}\right)=0$. For (3.7), notice that the Tanaka-Webster connection $\nabla$ satisfies $\nabla_{T} T=0$, and also $\tau T=0$ and $J T=0$, so that $\Omega(T, T)=0, A(T, T)=0,(\tau \otimes \theta)(T, T)=0(\theta \odot J)(T, T)=0$ which imply (3.7). 
Let us consider the generalized B.-Y. Chen's conjecture for pseudo biharmonic maps which is $\mathrm{CR}$ analogue of the usual generalized Chen's conjecture for biharmonic maps:

The $C R$ analogue of the generalized B.-Y. Chen's conjecture for pseudo biharmonic maps:

Let $\left(M, g_{\theta}\right)$ be a complete strictly pseudoconvex $C R$ manifold, and assume that $(N, h)$ is a Riemannian manifold of non-positive curvature.

Then, every pseudo biharmonic isometric immersion $\varphi:\left(M, g_{\theta}\right) \rightarrow(N, h)$ must be pseudo harmonic.

Then, we will show:

Theorem 2.1. Assume that $\varphi$ is a pseudo biharmonic map of a strictly pseudoconvex complete $C R$ manifold $\left(M, g_{\theta}\right)$ into another Riemannian manifold $(N, h)$ of non positive curvature.

If $\varphi$ has finite pseudo bienergy $E_{b, 2}(\varphi)<\infty$ and finite pseudo energy $E_{b}(\varphi)<\infty$, then it is pseudo harmonic, i.e., $\tau_{b}(\varphi)=0$.

\subsection{The Green's Formula on a Foliated Riemannian Manifold}

Then, we prepare the materials for the first and second variational formulas for the transversal energy of a smooth foliated map between two foliated Riemannian manifolds following [31] [32] [36]. Let $(M, g, \mathcal{F})$ be an $n(=p+q)$-dimensional foliated Riemannian manifold with foliation $\mathcal{F}$ of codimension $q$ and a bundle-like Riemannian metric g with respect to $\mathcal{F}$ (cf. [37] [38]). Let $T M$ be the tangent bundle of $M, L$, the tangent bundle of $\mathcal{F}$, and $Q=T M L$, the corresponding normal bundle of $\mathcal{F}$. We denote $g_{Q}$ the induced Riemannian metric on the normal bundle $Q$, and $\nabla^{Q}$, the transversal Levi-Civita connection on $Q, R^{Q}$, the transversal curvature tensor, and $K^{Q}$, the transversal sectional curvature, respectively. Notice that the bundle projection $\pi: T M \rightarrow Q$ is an element of the space $\Omega^{1}(M, Q)$ of $Q$-valued 1-forms on $M$. Then, one can obtain the $Q$-valued bilinear form $\alpha$ on $M$, called the second fundamental form of $\mathcal{F}$, defined by

$$
\alpha(X, Y)=-\left(D_{X} \pi\right)(Y)=\pi\left(\nabla_{X}^{Q} Y\right), \quad(X, Y \in \Gamma(L)) .
$$

The trace $\tau$ of $\alpha$, called the tension field of $\mathcal{F}$ is defined by

$$
\tau=\sum_{i, j=1}^{p} g^{i j} \alpha\left(X_{i}, X_{j}\right),
$$

where $\left\{X_{i}\right\}_{i=1}^{p}$ spanns $\Gamma(L \mid U)$ on a neighborhood $U$ on $M$. The Green's theorem, due to Yorozu and Tanemura [36], of a foliated Riemannian manifold $(M, g, \mathcal{F})$ says that

$$
\int_{M} \operatorname{div}_{D}(v) v_{g}=\int_{M} g_{Q}(\tau, v) v_{g} \quad(v \in \Gamma(Q)),
$$

where $\operatorname{div}_{D}(v)$ denotes the transversal divergence of $v$ with respect to $\nabla^{Q}$ given by $\operatorname{div}_{D}(v):=\sum_{a, b=1}^{q} g^{a b} g_{Q}\left(D_{X_{a}} v, \pi\left(X_{b}\right)\right)$. Here $\left\{X_{a}\right\}_{a=1}^{q}$ spanns $\Gamma\left(L^{\perp} \mid U\right)$ where $L^{\perp}$ is the orthogonal complement bundle of $L$ with a natural identification $\sigma: Q \stackrel{\cong}{\rightarrow} L^{\perp}$. 


\subsection{The Variational Formulas for Foliations}

Let $(M, g, \mathcal{F})$, and $\left(M^{\prime}, g^{\prime}, \mathcal{F}^{\prime}\right)$ be two compact foliated Riemannian manifolds. The transversal energy $E(\varphi)$ among the totality of smooth foliated maps from $(M, g, \mathcal{F})$ into $\left(M^{\prime}, g^{\prime}, \mathcal{F}^{\prime}\right)$ by

$$
E(\varphi)=\frac{1}{2} \int_{M}\left|d_{T} \varphi\right|^{2} v_{g} .
$$

Here, a smooth map $\varphi$ is a foliated map is, by definition, for every leaf $L$ of $\mathcal{F}$, there exists a leaf $L^{\prime}$ of $\mathcal{F}^{\prime}$ satisfying $\varphi(L) \subset L^{\prime}$. Then, $d_{T} \varphi:=\pi^{\prime} \circ d \varphi \circ \sigma ; Q \rightarrow Q^{\prime}$ can be regarded as a section of $Q^{*} \otimes \varphi^{-1} Q^{\prime}$ where $Q^{*}$ is a subspace of the cotangent bundle $T^{*} M$. Here, $\pi, \pi^{\prime}$ are the projections of $T M \rightarrow Q=T M L$ and $T M^{\prime} \rightarrow Q^{\prime}=T M^{\prime} / L^{\prime}$. Notice that our definition of the transversal energy is a slightly different from the one of Jung's definition (cf. [32], p. 5).

The first variational formula is given (cf. [?]), for every smooth foliated variation $\left\{\varphi_{t}\right\}$ with $\varphi_{0}=\varphi$ and $\left.\frac{\mathrm{d} \varphi_{t}}{\mathrm{~d} t}\right|_{t=0}=V$ in which $V$ being a section $\varphi^{-1} Q^{\prime}$,

$$
\left.\frac{\mathrm{d}}{\mathrm{d} t}\right|_{t=0} E\left(\varphi_{t}\right)=-\int_{M}\left\langle V, \tau_{b}(\varphi)\right\rangle v_{g}
$$

Here, $\tau_{b}(\varphi)$ is the transversal tension field defined by

$$
\tau_{b}(\varphi)=\sum_{a=1}^{q}\left(\tilde{\nabla}_{E_{a}} d_{T} \varphi\right)\left(E_{a}\right),
$$

where $\tilde{\nabla}$ is the induced connection in $Q^{*} \otimes \varphi^{-1} Q^{\prime}$ from the Levi-Civita connection of $\left(M^{\prime}, g^{\prime}\right)$, and $\left\{E_{a}\right\}_{a=1}^{q}$ is a locally defined orthonormal frame field on $Q$.

Definition 2.2. A smooth foliated map $\varphi:(M, g, \mathcal{F}) \rightarrow\left(M^{\prime}, g^{\prime}, \mathcal{F}^{\prime}\right)$ is said to be transversally harmonic if $\tau_{b}(\varphi) \equiv 0$.

Then, for a transversally harmonic map $\varphi:(M, g, \mathcal{F}) \rightarrow\left(M^{\prime}, g^{\prime}, \mathcal{F}^{\prime}\right)$, the second variation formula of the transversal energy $E(\varphi)$ is given as follows (cf. [?], p. 7) : let $\varphi_{s, t}: M \rightarrow M^{\prime} \quad(-\epsilon<s, t<\epsilon)$ be any two parameter smooth foliated variation of $\varphi$ with $V=\left.\frac{\partial \varphi_{s, t}}{\partial s}\right|_{(s, t)=(0,0)}, W=\left.\frac{\partial \varphi_{s, t}}{\partial t}\right|_{(s, t)=(0,0)}$ and $\varphi_{0,0}=\varphi$,

$$
\begin{aligned}
\operatorname{Hess}(E)_{\varphi}(V, W) & :=\left.\frac{\partial^{2}}{\partial s \partial t}\right|_{(s, t)=(0,0)} E\left(\varphi_{s, t}\right) \\
& =\int_{M}\left\langle J_{b, \varphi}(V), W\right\rangle v_{g},
\end{aligned}
$$

where $J_{b, \varphi}$ is a second order semi-elliptic differential operator acting on the space $\Gamma\left(\varphi^{-1} Q^{\prime}\right)$ of sections of $\varphi^{-1} Q^{\prime}$ which is of the form:

$$
\begin{aligned}
J_{b, \varphi}(V) & :=\tilde{\nabla}^{*} \tilde{\nabla} V-\operatorname{trace}_{Q} R^{Q^{\prime}}\left(V, d_{T} \varphi\right) d_{T} \varphi \\
& =-\sum_{a=1}^{q}\left(\tilde{\nabla}_{E_{a}} \tilde{\nabla}_{E_{a}}-\tilde{\nabla}_{\nabla_{E_{a}} E_{a}}\right) V-\sum_{a=1}^{q} R^{Q^{\prime}}\left(V, d_{T} \varphi\left(E_{a}\right)\right) d_{T} \varphi\left(E_{a}\right)
\end{aligned}
$$

for $V \in \Gamma\left(\varphi^{-1} Q^{\prime}\right)$. Here, $\nabla$ is the Levi-Civita connection of $(M, g)$, and recall also that: 


$$
\begin{aligned}
& \tilde{\nabla}^{*} \tilde{\nabla} V:=-\sum_{a=1}^{q}\left(\tilde{\nabla}_{E_{a}} \tilde{\nabla}_{E_{a}}-\tilde{\nabla}_{\nabla_{E_{a} E_{a}}}\right) V, \\
& \operatorname{trace}_{Q} R^{Q^{\prime}}\left(V, d_{T} \varphi\right) d_{T} \varphi:=\sum_{a=1}^{q} R^{Q^{\prime}}\left(V, d_{T} \varphi\left(E_{a}\right)\right) d_{T} \varphi\left(E_{a}\right) .
\end{aligned}
$$

Definition 2.3. The transversal bitension field $\tau_{2, b}(\varphi)$ of a smooth foliated map $\varphi$ is defined by

$$
\tau_{2, b}(\varphi):=J_{b, \varphi}\left(\tau_{b}(\varphi)\right)
$$

Definition 2.4. The transversal bienergy $E_{2}$ of a smooth foliated map $\varphi$ is defined by

$$
E_{2}(\varphi):=\frac{1}{2} \int_{M}\left|\tau_{b}(\varphi)\right|^{2} v_{g}
$$

Remark that this definition of the transversal bienergy is also slightly different from the one of Jung (cf. Jung [32], p. 13, Definition 6.1). On the first variation formula of the transversal bienergy is given as follows. For a smooth foliated map $\varphi$ and a smooth foliated variation $\left\{\varphi_{t}\right\}$ of $\varphi$, it holds (cf. [32], p. 13) that

$$
\left.\frac{\mathrm{d}}{\mathrm{d} t}\right|_{t=0} E_{2}\left(\varphi_{t}\right)=-\int_{M}\left\langle V, \tau_{2, b}(\varphi)\right\rangle v_{g} .
$$

Definition 2.5. A smooth foliated map $\varphi:(M, g, \mathcal{F}) \rightarrow\left(M^{\prime}, g^{\prime}, \mathcal{F}^{\prime}\right)$ is said to be transversally biharmonic if $\tau_{2, b}(\varphi) \equiv 0$.

Then, one can ask the following generalized B.Y. Chen's conjecture:

The generalized Chen's conjecture:

Let $\varphi$ be a transversally biharmonic map from a foliated Riemannian manifold $(M, g, \mathcal{F})$ into another foliated Riemannian manifold $\left(M^{\prime}, g^{\prime}, \mathcal{F}^{\prime}\right)$ whose transversal sectional curvature $K^{Q^{\prime}}$ is non-positive. Then, $\varphi$ must be transversally harmonic.

Then, we can state our main theorem which gives an affirmative partial answer to the above generalized Chen's conjecture under the additional assumption that $\varphi$ has both the finite transversal energy and the finite transversal bienergy:

Theorem 2.6. Let $\varphi:(M, g, \mathcal{F}) \rightarrow\left(M^{\prime}, g^{\prime}, \mathcal{F}^{\prime}\right)$ a smooth foliated map which is an isometric immersion of $(M, g)$ into $\left(M^{\prime}, g^{\prime}\right)$. Assume that $(M, g)$ is complete (possibly non-compact), and the transversal sectional curvature $K^{Q^{\prime}}$ of $\left(M^{\prime}, g^{\prime}, \mathcal{F}^{\prime}\right)$ is non-positive.

If $\varphi$ is transversally biharmonic having both the finite transversal energy $E(\varphi)<\infty$ and the finite transversal bienergy $E_{2}(\varphi)$, then it is transversally harmonic.

Remark that in the case that $M$ is compact, Theorem 2.5 is true due to Jung's work (cf. [32] Theorem 6.4, p. 14).

\section{Proof of Theorem 2.1}

The proof of Theorem 2.1 is divided into several steps which will appear in [20].

(The first step) For an arbitrarily fixed point $x_{0} \in M$, let $B_{r}\left(x_{0}\right)=\{x \in M: r(x)<r\}$ where $r(x)$ is a distance function on $\left(M, g_{\theta}\right)$, and let us take a cut off function $\eta$ 
on $\left(M, g_{\theta}\right)$, i.e.,

$$
\left\{\begin{array}{lc}
0 \leq \eta(x) \leq 1 & (x \in M), \\
\eta(x)=1 & \left(x \in B_{r}\left(x_{0}\right)\right), \\
\eta(x)=0 & \left(x \notin B_{2 r}\left(x_{0}\right)\right), \\
\left|\nabla^{g_{\theta}} \eta\right| \leq \frac{2}{r} & (x \in M),
\end{array}\right.
$$

where $r$ is the distance function from $x_{0} \in M$, and $\nabla^{g_{\theta}}$ is the Levi-Civita connection of $\left(M, g_{\theta}\right)$, respectively. Assume that $\varphi:\left(M, g_{\theta}\right) \rightarrow(N, h)$ is a pseudo biharmonic map, i.e.,

$$
\begin{aligned}
\tau_{b, 2}(\varphi) & =J_{b}\left(\tau_{b}(\varphi)\right) \\
& =\Delta_{b}\left(\tau_{b}(\varphi)\right)-\sum_{j=1}^{2 n} R^{h}\left(\tau_{b}(\varphi), \mathrm{d} \varphi\left(X_{j}\right)\right) \mathrm{d} \varphi\left(X_{j}\right) \\
& =0 .
\end{aligned}
$$

(The second step) Then, we have

$$
\begin{aligned}
& \int_{M}\left\langle\Delta_{b}\left(\tau_{b}(\varphi)\right), \eta^{2} \tau_{b}(\varphi)\right\rangle \theta \wedge(\mathrm{d} \theta)^{n} \\
& =\int_{M} \eta^{2} \sum_{j=1}^{2 n}\left\langle R^{h}\left(\tau_{b}(\varphi), \mathrm{d} \varphi\left(X_{j}\right)\right) \mathrm{d} \varphi\left(X_{j}\right), \tau_{b}(\varphi)\right\rangle \theta \wedge(\mathrm{d} \theta)^{n} .
\end{aligned}
$$

In (3.3), notice that $\left\langle R^{h}\left(\tau_{b}(\varphi), \mathrm{d} \varphi\left(X_{j}\right)\right) \mathrm{d} \varphi\left(X_{j}\right), \tau_{b}(\varphi)\right\rangle$ is the sectional curvature of $(N h)$ corresponding to the vectors $\tau_{b}(\varphi)$ and $\mathrm{d} \varphi\left(X_{j}\right)$. Since $(N, h)$ has the non-positive sectional curvature, (3.3) is non-positive.

On the other hand, for the left hand side of (3.3), it holds that

$$
\begin{aligned}
& \int_{M}\left\langle\Delta_{b}\left(\tau_{b}(\varphi)\right), \eta^{2} \tau_{b}(\varphi)\right\rangle \theta \wedge(\mathrm{d} \theta)^{n} \\
= & \int_{M}\left\langle\bar{\nabla}^{H} \tau_{b}(\varphi), \bar{\nabla}^{H}\left(\eta^{2} \tau_{b}(\varphi)\right)\right\rangle \theta \wedge(\mathrm{d} \theta)^{n} \\
= & \int_{M} \sum_{j=1}^{2 n}\left\langle\bar{\nabla}_{X_{j}} \tau_{b}(\varphi), \bar{\nabla}_{X_{j}}\left(\eta^{2} \tau_{b}(\varphi)\right)\right\rangle \theta \wedge(\mathrm{d} \theta)^{n} .
\end{aligned}
$$

Here, let us recall, for $V, W \in \Gamma\left(\varphi^{-1} T N\right)$,

$$
\left\langle\bar{\nabla}^{H} V, \bar{\nabla}^{H} W\right\rangle=\sum_{\alpha}\left\langle\bar{\nabla}_{e_{\alpha}}^{H} V, \bar{\nabla}_{e_{\alpha}}^{H} W\right\rangle=\sum_{j=1}^{2 n}\left\langle\bar{\nabla}_{X_{i}} V, \bar{\nabla}_{X_{i}} W\right\rangle,
$$

where $\left\{e_{\alpha}\right\}$ is a locally defined orthonormal frame field of $\left(M, g_{\theta}\right)$ and $\bar{\nabla}_{X}^{H} W$ $\left(X \in \mathfrak{X}(M), W \in \Gamma\left(\varphi^{-1} T N\right)\right)$ is defined by

$$
\bar{\nabla}_{X}^{H} W=\sum_{j}\left\{\left(X^{H} f_{j}\right) V_{j}+f_{j} \bar{\nabla}_{X^{H}} V_{j}\right\}
$$

for $W=\sum_{j} f_{i} V_{j} \quad\left(f_{j} \in C^{\infty}(M)\right)$ and $V_{j} \in \Gamma\left(\varphi^{-1} T N\right)$. Here, $X^{H}$ is the $H(M)$ -component of $X$ corresponding to the decomposition of $T_{x}(M)=H_{x}(M) \oplus \mathbb{R} T_{x}$ $(x \in M)$, and $\bar{\nabla}$ is the induced connection of $\varphi^{-1} T N$ from the Levi-Civita connection $\nabla^{h}$ of $(N, h)$.

Since 


$$
\bar{\nabla}_{X_{j}}\left(\eta^{2} \tau_{b}(\varphi)\right)=2 \eta X_{j} \eta \tau_{b}(\varphi)+\eta^{2} \bar{\nabla}_{X_{j}} \tau_{b}(\varphi),
$$

the right hand side of (3.4) is equal to

$$
\int_{M} \eta^{2} \sum_{j=1}^{2 n}\left|\bar{\nabla}_{X_{j}} \tau_{b}(\varphi)\right|^{2} \theta \wedge(\mathrm{d} \theta)^{n}+2 \int_{M} \sum_{j=1}^{2 n}\left\langle\eta \bar{\nabla}_{X_{j}} \tau_{b}(\varphi),\left(X_{j} \eta\right) \tau_{b}(\varphi)\right\rangle \theta \wedge(\mathrm{d} \theta)^{n} .
$$

Therefore, together with (3.3), we have

$$
\begin{aligned}
& \int_{M} \eta^{2} \sum_{j=1}^{2 n}\left|\bar{\nabla}_{X_{j}} \tau_{b}(\varphi)\right|^{2} \theta \wedge(\mathrm{d} \theta)^{n} \\
& \leq-2 \int_{M} \sum_{j=1}^{2 n}\left\langle\eta \bar{\nabla}_{X_{j}} \tau_{b}(\varphi),\left(X_{j} \eta\right) \tau_{b}(\varphi)\right\rangle \theta \wedge(\mathrm{d} \theta)^{n} \\
& =:-2 \int_{M} \sum_{j=1}^{2 n}\left\langle V_{j}, W_{j}\right\rangle \theta \wedge(\mathrm{d} \theta)^{n},
\end{aligned}
$$

where we define $V_{j}, W_{j} \in \Gamma\left(\varphi^{-1} T N\right) \quad(j=1, \cdots, 2 n)$ by

$$
V_{j}:=\eta \bar{\nabla}_{X_{j}} \tau_{b}(\varphi), \quad W_{j}:=\left(X_{j} \eta\right) \tau_{b}(\varphi) .
$$

Then, it holds that $0 \leq\left|\sqrt{\epsilon} V_{i} \pm \frac{1}{\sqrt{\epsilon}} W_{i}\right|^{2}$ for every $\epsilon>0$ which implies that

$$
\mp 2\left\langle V_{i}, W_{i}\right\rangle \leq \epsilon\left|V_{i}\right|^{2}+\frac{1}{\epsilon}\left|W_{i}\right|^{2} \text {. }
$$

Therefore, we have that

The right hand side of (3.7)

$$
\leq \epsilon \int_{M} \sum_{j=1}^{2 n}\left|V_{j}\right|^{2} \theta \wedge(\mathrm{d} \theta)^{n}+\frac{1}{\epsilon} \int_{M} \sum_{j=1}^{2 n}\left|W_{j}\right|^{2} \theta \wedge(\mathrm{d} \theta)^{n}
$$

foe every $\epsilon>0$. By taking $\epsilon=\frac{1}{2}$, we obtain

$$
\begin{aligned}
& \int_{M} \eta^{2} \sum_{j=1}^{2 n}\left|\bar{\nabla}_{X_{j}} \tau_{b}(\varphi)\right|^{2} \theta \wedge(\mathrm{d} \theta)^{n} \\
& \leq \frac{1}{2} \int_{M} \sum_{j=1}^{2 n} \eta^{2}\left|\bar{\nabla}_{X_{j}} \tau_{b}(\varphi)\right|^{2} \theta \wedge(\mathrm{d} \theta)^{n}+2 \int_{M} \sum_{j=1}^{2 n}\left|X_{j} \eta\right|^{2}\left|\tau_{b}(\varphi)\right|^{2} \theta \wedge(\mathrm{d} \theta)^{n} .
\end{aligned}
$$

Therefore, we obtain, due to the properties that $\eta=1$ on $B_{r}\left(x_{0}\right)$, and

$$
\begin{aligned}
\sum_{j=1}^{2 n}\left|X_{j} \eta\right|^{2} \leq\left|\nabla^{g_{\theta}} \eta\right|^{2} \leq\left(\frac{2}{r}\right)^{2}, & \\
\int_{B_{r}\left(x_{0}\right)} \sum_{j=1}^{2 n}\left|\bar{\nabla} \tau_{b}(\varphi)\right|^{2} \theta \wedge(\mathrm{d} \theta)^{n} & \leq \int_{M} \eta^{2} \sum_{j=1}^{2 n}\left|\bar{\nabla}_{X_{j}} \tau_{b}(\varphi)\right|^{2} \theta \wedge(\mathrm{d} \theta)^{n} \\
& \leq 4 \int_{M} \sum_{j=1}^{2 n}\left|X_{j} \eta\right|^{2}\left|\tau_{b}(\varphi)\right|^{2} \theta \wedge(\mathrm{d} \theta)^{n} \\
& \leq \frac{16}{r^{2}} \int_{M}\left|\tau_{b}(\varphi)\right|^{2} \theta \wedge(\mathrm{d} \theta)^{n} .
\end{aligned}
$$

(The third step) By our assumption that $E_{b, 2}(\varphi)=\frac{1}{2} \int_{M}\left|\tau_{b}(\varphi)\right|^{2} \theta \wedge(d \theta)^{n}<\infty$ and 
$\left(M, g_{\theta}\right)$ is complete, if we let $r \rightarrow \infty$, then $B_{r}\left(x_{0}\right)$ goes to $M$, and the right hand side of (3.10) goes to zero. We have

$$
\int_{M} \sum_{j=1}^{2 n}\left|\bar{\nabla}_{X_{j}} \tau_{b}(\varphi)\right|^{2} \theta \wedge(\mathrm{d} \theta)^{n}=0
$$

This implies that

$$
\left.\bar{\nabla}_{X} \tau_{b}(\varphi)=0 \quad \text { (for all } X \in H(M)\right) .
$$

(The fourth step) Let us take a 1 form $\alpha$ on $M$ defined by

$$
\alpha(X)= \begin{cases}\left\langle\mathrm{d} \varphi(X), \tau_{b}(\varphi)\right\rangle, & (X \in H(M)), \\ 0, & (X=T) .\end{cases}
$$

Then, we have

$$
\begin{aligned}
\int_{M}|\alpha| \theta \wedge(\mathrm{d} \theta)^{n} & =\int_{M}\left(\sum_{j=1}^{2 n}\left|\alpha\left(X_{j}\right)\right|^{2}\right)^{\frac{1}{2}} \theta \wedge(\mathrm{d} \theta)^{n} \\
& \leq\left(\left|d_{b} \varphi\right|^{2} \theta \wedge(\mathrm{d} \theta)^{n}\right)^{\frac{1}{2}}\left(\int_{M}\left|\tau_{b}(\varphi)\right|^{2} \theta \wedge(\mathrm{d} \theta)^{n}\right)^{\frac{1}{2}} \\
& =2 \sqrt{E_{b}(\varphi) E_{b, 2}(\varphi)}<\infty,
\end{aligned}
$$

where we put $d_{b} \varphi:=\sum_{i=1}^{2 n} \mathrm{~d} \varphi\left(X_{i}\right) \otimes X_{i}$,

$$
\left|d_{b} \varphi\right|^{2}=\sum_{i, j=1}^{2 n} g_{\theta}\left(X_{i}, X_{j}\right) h\left(\mathrm{~d} \varphi\left(X_{i}\right), \mathrm{d} \varphi\left(X_{j}\right)\right)=\sum_{i=1}^{2 n} h\left(\mathrm{~d} \varphi\left(X_{i}\right), \mathrm{d} \varphi\left(X_{i}\right)\right),
$$

and

$$
E_{b}(\varphi)=\frac{1}{2} \int_{M}\left|d_{b} \varphi\right|^{2} \theta \wedge(\mathrm{d} \theta)^{n} .
$$

Furthermore, let us define a $C^{\infty}$ function $\delta_{b} \alpha$ on $M$ by

$$
\delta_{b} \alpha=-\sum_{j=1}^{2 n}\left(\nabla_{X_{j}} \alpha\right)\left(X_{j}\right)=-\sum_{j=1}^{2 n}\left\{X_{j}\left(\alpha\left(X_{j}\right)\right)-\alpha\left(\nabla_{X_{j}} X_{j}\right)\right\},
$$

where $\nabla$ is the Tanaka-Webster connection. Notice that

$$
\begin{aligned}
\operatorname{div}(\alpha) & =\sum_{j=1}^{2 n}\left(\nabla_{X_{j}}^{g_{\theta}} \alpha\right)\left(X_{j}\right)+\left(\nabla_{T}^{g_{\theta}} \alpha\right)(T) \\
& =\sum_{j=1}^{2 n}\left\{X_{j}\left(\alpha \circ \pi_{H}\left(X_{j}\right)\right)-\alpha \circ \pi_{H}\left(\nabla_{X_{j}}^{g_{\theta}} X_{j}\right)\right\}+T\left(\alpha \circ \pi_{H}(T)\right)-\alpha \circ \pi_{H}\left(\nabla_{T}^{g_{\theta}} T\right) \\
& =\sum_{j=1}^{2 n}\left\{X_{j}\left(\alpha\left(X_{j}\right)\right)-\alpha\left(\pi_{H}\left(\nabla_{X_{j}}^{g_{\theta}} X_{j}\right)\right)\right\} \\
& =\sum_{j=1}^{2 n}\left\{X_{j}\left(\alpha\left(\left(X_{j}\right)\right)-\alpha\left(\nabla_{X_{j}} X_{j}\right)\right\}\right. \\
& =-\delta_{b} \alpha,
\end{aligned}
$$

where $\pi_{H}: T_{\chi}(M) \rightarrow H_{\chi}(M)$ is the natural projection. We used the facts that $\nabla_{T}^{g_{\theta}} T=0$, and $\pi_{H}\left(\nabla_{X}^{g_{\theta}} Y\right)=\nabla_{X} Y \quad(X, Y \in H(M))$ ([35], p.37). Here, recall again $\nabla^{g_{\theta}}$ is the Levi-Civita connection of $g_{\theta}$, and $\nabla$ is the Tanaka-Webster connection. 
Then, we have, for (3.16),

$$
\begin{aligned}
\delta_{b} \alpha & =-\sum_{j=1}^{2 n}\left\{X_{j}\left\langle\mathrm{~d} \varphi\left(X_{j}\right), \tau_{b}(\varphi)\right\rangle-\left\langle\mathrm{d} \varphi\left(\nabla_{X_{j}} X_{j}\right), \tau_{b}(\varphi)\right\rangle\right\} \\
& =-\sum_{j=1}^{2 n}\left\{\left\langle\bar{\nabla}_{X_{j}}\left(\mathrm{~d} \varphi\left(X_{j}\right)\right), \tau_{b}(\varphi)\right\rangle+\left\langle\mathrm{d} \varphi\left(X_{j}\right), \bar{\nabla}_{X_{j}} \tau_{b}(\varphi)\right\rangle-\left\langle\mathrm{d} \varphi\left(\nabla_{X_{j}} X_{j}\right), \tau_{b}(\varphi)\right\rangle\right\} \\
& =-\left\langle\sum_{j=1}^{2 n}\left\{\bar{\nabla}_{X_{j}}\left(\mathrm{~d} \varphi\left(X_{j}\right)\right)-\mathrm{d} \varphi\left(\nabla_{X_{j}} X_{j}\right)\right\}, \tau_{b}(\varphi)\right\rangle \\
& =-\left|\tau_{b}(\varphi)\right|^{2} .
\end{aligned}
$$

We used (3.12) $\bar{\nabla}_{X_{j}} \tau_{b}(\varphi)=0$ to derive the last second equality of (3.17). Then, due to (3.17), we have for $E_{b, 2}(\varphi)$,

$$
\begin{aligned}
E_{b, 2}(\varphi) & =\frac{1}{2} \int_{M}\left|\tau_{b}(\varphi)\right|^{2} \theta \wedge(\mathrm{d} \theta)^{n} \\
& =-\frac{1}{2} \int_{M} \delta_{b} \alpha \theta \wedge(\mathrm{d} \theta)^{n} \\
& =\frac{1}{2} \int_{M} \operatorname{div}(\alpha) \theta \wedge(\mathrm{d} \theta)^{n} \\
& =0 .
\end{aligned}
$$

In the last equality, we used Gaffney's theorem ([16], p. 271, [?]).

Therefore, we obtain $\tau_{b}(\varphi) \equiv 0$, i.e., $\varphi$ is pseudo harmonic.

We obtain Theorem 2.1 .

\section{Proof of Main Theorem 2.6}

In this section, we give a proof of Theorem 2.6 which will appear in [34], by a similar way to the case of foliations as Theorem 2.1.

(The first step) First, let us take a cut off function $\eta$ from a fixed point $x_{0} \in M$ on $(M, g)$, i.e.,

$$
\left\{\begin{array}{lr}
0 \leq \eta(x) \leq 1 & (x \in M), \\
\eta(x)=1 & \left(x \in B_{r}\left(x_{0}\right)\right), \\
\eta(x)=0 & \left(x \notin B_{2 r}\left(x_{0}\right)\right), \\
\left|\nabla^{g} \eta\right| \leq \frac{2}{r} & (x \in M),
\end{array}\right.
$$

where $B_{r}\left(x_{0}\right):=\{x \in M \mid r(x)<r\}, r(x)$ is a distance function from $x_{0}$ on $(M, g)$, $\nabla^{g}$ is the Levi-Civita connection of $(M, g)$, respectively.

Assume that $\varphi$ is a transversally biharmonic map of $(M, g, F)$ into $\left(M^{\prime}, g^{\prime}, F^{\prime}\right)$, i.e.,

$$
\begin{aligned}
J_{2, b}(\varphi) & =J_{b, \varphi}\left(\tau_{b}(\varphi)\right) \\
& =\tilde{\nabla} \tilde{\nabla}^{*} \tau_{b}(\varphi)-\operatorname{trace}_{Q} R^{Q^{\prime}}\left(\tau_{b}(\varphi), d_{T} \varphi\right) d_{T} \varphi \\
& =0,
\end{aligned}
$$

where recall $\tilde{\nabla}$ is the induced connection on $\varphi^{-1} Q^{\prime} \otimes T^{*} M$.

(The second step) Then, by (4.1), we obtain that 


$$
\begin{aligned}
\int_{M}\left\langle\tilde{\nabla}^{*} \tilde{\nabla} \tau_{b}(\varphi), \eta^{2} \tau_{b}(\varphi)\right\rangle v_{g} & =\int_{M} \eta^{2}\left\langle\operatorname{trace}_{Q} R^{Q^{\prime}}\left(\tau_{b}(\varphi), d_{T} \varphi\right) d_{T} \varphi, \tau_{b}(\varphi)\right\rangle v_{g} \\
& =\int_{M} \eta^{2} \sum_{a=1}^{q}\left\langle R^{Q^{\prime}}\left(\tau_{b}(\varphi), d_{T} \varphi\left(E_{a}\right)\right) d_{T} \varphi\left(E_{a}\right), \tau_{b}(\varphi)\right\rangle v_{g} \\
& =\int_{M} \eta^{2} \sum_{a=1}^{q} K^{Q^{\prime}}\left(\Pi_{\varphi, a}\right) v_{g} \\
& \leq 0,
\end{aligned}
$$

where the sectional curvature $K^{Q^{\prime}}\left(\Pi_{\varphi, a}\right)$ of $\left(M^{\prime}, g^{\prime}, \mathcal{F}^{\prime}\right)$ corresponding to the plane spanned by $\tau_{b}(\varphi)$ and $d_{T} \varphi\left(E_{a}\right)$ is non-positive.

(The third step) On the other hand, the left hand side of (4.2) is equal to

$$
\begin{aligned}
& \int_{M}\left\langle\tilde{\nabla} \tau_{b}(\varphi), \tilde{\nabla}\left(\eta^{2} \tau_{b}(\varphi)\right)\right\rangle v_{g} \\
& =\int_{M} \sum_{a=1}^{q}\left\langle\tilde{\nabla}_{E_{a}} \tau_{b}(\varphi), \tilde{\nabla}_{E_{a}}\left(\eta^{2} \tau_{b}(\varphi)\right)\right\rangle v_{g} \\
& =\int_{M} \eta^{2} \sum_{a=1}^{q}\left|\tilde{\nabla}_{E_{a}} \tau_{b}(\varphi)\right|^{2} v_{g}+2 \int_{M} \sum_{a=1}^{q}\left\langle\eta \tilde{\nabla}_{E_{a}} \tau_{b}(\varphi),\left(E_{a} \eta\right) \tau_{b}(\varphi)\right\rangle v_{g}
\end{aligned}
$$

since

$$
\tilde{\nabla}_{E_{a}}\left(\eta^{2} \tau_{b}(\varphi)\right)=\eta^{2} \tilde{\nabla}_{E_{a}} \tau_{b}(\varphi)+2 \eta\left(E_{a} \eta\right) \tau_{b}(\varphi) .
$$

Together (4.2) and (4.3), we obtain

$$
\begin{aligned}
\int_{M} \eta^{2} \sum_{a=1}^{q}\left|\tilde{\nabla} \tau_{b}(\varphi)\right|^{2} v_{g} & \leq-2 \int_{M} \sum_{a=1}^{q}\left\langle\eta \tilde{\nabla}_{E_{a}} \tau_{b}(\varphi),\left(E_{a} \eta\right) \tau_{b}(\varphi)\right\rangle v_{g} \\
& \leq \frac{1}{2} \int_{M} \eta^{2} \sum_{a=1}^{q}\left|\tilde{\nabla}_{E_{a}} \tau_{b}(\varphi)\right|^{2} v_{g}+2 \int_{M} \sum_{a=1}^{q}\left|E_{a} \eta\right|^{2}\left|\tau_{b}(\varphi)\right|^{2} v_{g} .
\end{aligned}
$$

Because, putting $V_{a}:=\eta \tilde{\nabla}_{E_{a}} \tau_{b}(\varphi), W_{a}:=\left(E_{a} \eta\right) \tau_{b}(\eta) \quad(a=1, \cdots, q)$, we have

$$
0 \leq\left|\sqrt{\epsilon} V_{a} \pm \frac{1}{\sqrt{\epsilon}} W_{a}\right|^{2}=\epsilon\left|V_{a}\right|^{2} \pm 2\left\langle V_{a}, W_{a}\right\rangle+\frac{1}{\epsilon}\left|W_{a}\right|^{2}
$$

which is

$$
\mp 2\left\langle V_{a}, W_{a}\right\rangle \leq \epsilon\left|V_{a}\right|^{2}+\frac{1}{\epsilon}\left|W_{a}\right|^{2} .
$$

If we put $\epsilon=\frac{1}{2}$ in (4.5), then we obtain

$$
\mp 2\left\langle V_{a}, W_{a}\right\rangle \leq \frac{1}{2}\left|V_{a}\right|^{2}+2\left|W_{a}\right|^{2} \quad(a=1, \cdots, q) .
$$

By (4.6), we have the second inequality of (4.4).

(The fourth step) Noticing that $\eta=1$ on $B_{r}\left(x_{0}\right)$ and $\left|E_{a} \eta\right|^{2} \leq \frac{2}{r}$ in the inequality (3.4), we obtain

$$
\begin{aligned}
\int_{B_{r}\left(x_{0}\right)} \sum_{a=1}^{q}\left|\tilde{\nabla}_{E_{a}} \tau_{b}(\varphi)\right|^{2} v_{g} & =\int_{B_{r}\left(x_{0}\right)} \eta^{2} \sum_{a=1}^{q}\left|\tilde{\nabla}_{E_{a}} \tau_{b}(\varphi)\right|^{2} v_{g} \leq \int_{M} \eta^{2} \sum_{a=1}^{q}\left|\tilde{\nabla}_{E_{a}} \tau_{b}(\varphi)\right|^{2} v_{g} \\
& \leq 4 \int_{M} \sum_{a=1}^{q}\left|E_{a} \eta\right|^{2}\left|\tau_{b}(\varphi)\right|^{2} v_{g} \leq \frac{16}{r^{2}} \int_{M}\left|\tau_{b}(\varphi)\right|^{2} v_{g} .
\end{aligned}
$$


Letting $r \rightarrow \infty$, the right hand side of (4.7) converges to zero since

$E_{2}(\varphi)=\frac{1}{2} \int_{M}\left|\tau_{b}(\varphi)\right|^{2} v_{g}<\infty$. But due to (4.7), the left hand side of (4.7) must converge to $\int_{M} \sum_{a=1}^{q}\left|\tilde{\nabla}_{E_{a}} \tau_{b}(\varphi)\right|^{2} v_{g}$ since $B_{r}\left(X_{0}\right)$ tends to $M$ because $(M, g)$ is complete. Therefore, we obtain that

$$
0 \leq \int_{M} \sum_{a=1}^{q}\left|\tilde{\nabla}_{E_{a}} \tau_{b}(\varphi)\right|^{2} v_{g} \leq 0,
$$

which implies that

$$
\tilde{\nabla}_{E_{a}} \tau_{b}(\varphi)=0(a=1, \cdots, q) \text {, i.e., } \tilde{\nabla}_{X} \tau_{b}(\varphi)=0(\forall X \in \Gamma(Q)) .
$$

(The fifth step) Let us define a 1-form $\alpha$ on $M$ by

$$
\alpha(X):=\left\langle\mathrm{d} \varphi(\pi(X)), \tau_{b}(\varphi)\right\rangle, \quad(X \in \mathfrak{X}(M)),
$$

and a canonical dual vector field $\alpha^{\#} \in \mathfrak{X}(M)$ on $M$ by $\left\langle\alpha^{\#}, Y\right\rangle:=\alpha(Y),(Y \in \mathfrak{X}(M))$. Then, its divergence $\operatorname{div}\left(\alpha^{\#}\right)$ written as

$$
\operatorname{div}\left(\alpha^{\#}\right)=\sum_{i=1}^{p} g\left(\nabla_{E_{i}}^{g} \alpha^{\#}, E_{i}\right)+\sum_{a=1}^{q} g\left(\nabla_{E_{a}}^{g} \alpha^{\#}, E_{a}\right),
$$

can be given as follows. Here, $\left\{E_{i}\right\}_{i=1}^{p}$ and $\left\{E_{a}\right\}_{a=1}^{q}$ are locally defined orthonormal frame fields on leaves $L$ of $\mathcal{F}$ and $Q$, respectively, $\left(\operatorname{dim} L_{x}=p, \operatorname{dim} Q_{x}=q, x \in M\right)$.

Then, we can calculate $\operatorname{div}\left(\alpha^{\#}\right)$ as follows:

$$
\begin{aligned}
\operatorname{div}\left(\alpha^{\#}\right)= & \sum_{i=1}^{p}\left\{E_{i}\left(\alpha\left(E_{i}\right)\right)-\alpha\left(\nabla_{E_{i}}^{g} E_{i}\right)\right\}+\sum_{a=1}^{q}\left\{E_{a}\left(\alpha\left(E_{a}\right)\right)-\alpha\left(\nabla_{E_{a}}^{g} E_{a}\right)\right\} \\
= & \left\langle\mathrm{d} \varphi\left(\pi\left(-\sum_{i=1}^{p} \nabla_{E_{i}}^{g} E_{i}\right)\right), \tau_{b}(\varphi)\right\rangle \\
& +\sum_{a=1}^{q}\left\{E_{a}\left\langle\mathrm{~d} \varphi\left(E_{a}\right), \tau_{b}(\varphi)\right\rangle-\left\langle\mathrm{d} \varphi\left(\pi\left(\nabla_{E_{a}}^{g} E_{a}\right)\right), \tau_{b}(\varphi)\right)\right\} \\
= & \left\langle\mathrm{d} \varphi\left(\pi\left(-\sum_{i=1}^{p} \nabla_{E_{i}}^{g} E_{i}\right)\right), \tau_{b}(\varphi)\right\rangle \\
& +\sum_{a=1}^{q}\left\{\left\langle\tilde{\nabla}_{E_{a}}\left(\mathrm{~d} \varphi\left(E_{a}\right)\right), \tau_{b}(\varphi)\right\rangle+\left\langle\mathrm{d} \varphi\left(E_{a}\right), \tilde{\nabla}_{E_{a}} \tau_{b}(\varphi)\right\rangle-\left\langle\mathrm{d} \varphi\left(\pi\left(\nabla_{E_{a}}^{g} E_{a}\right)\right), \tau_{b}(\varphi)\right\rangle\right\} \\
= & \left\langle\mathrm{d} \varphi\left(\pi\left(-\sum_{i=1}^{p} \nabla_{E_{i}}^{g} E_{i}\right)\right)+\sum_{a=1}^{q}\left\{\tilde{\nabla}_{E_{a}}\left(\mathrm{~d} \varphi\left(E_{a}\right)\right)-\mathrm{d} \varphi\left(\pi\left(\nabla_{E_{a}}^{g} E_{a}\right)\right)\right\}, \tau_{b}(\varphi)\right\rangle .
\end{aligned}
$$

since $\tilde{\nabla}_{E_{a}} \tau_{b}(\varphi)=0$ in the last equality of (4.10). Integrating the both hands of (4.10) over $M$, we have

$$
\begin{aligned}
& \int_{M}\left\langle\mathrm{~d} \varphi\left(\pi\left(\sum_{i=1}^{p} \nabla_{E_{i}}^{g} E_{i}\right)\right), \tau_{b}(\varphi)\right\rangle v_{g} \\
& =\int_{M}\left\langle\sum_{a=1}^{q}\left\{\tilde{\nabla}_{E_{a}}\left(\mathrm{~d} \varphi\left(E_{a}\right)\right)-\mathrm{d} \varphi\left(\pi\left(\nabla_{E_{a}}^{g} E_{a}\right)\right)\right\}, \tau_{b}(\varphi)\right\rangle v_{g} .
\end{aligned}
$$

because of $\int_{M} \operatorname{div}\left(\alpha^{\#}\right) v_{g}=0$. Notice that both hands in (4.11) are well defined because 
of $E(\varphi)<\infty$ and $E_{2}(\varphi)<\infty$.

Since $\kappa^{\#}:=\pi\left(\sum_{i=1}^{p} \nabla_{E_{i}}^{g} E_{i}\right)$ is the second fundamental form of each leaf $L$ in $(M, g)$ and

$$
\begin{aligned}
\tau_{b}(\varphi) & =\sum_{a=1}^{q}\left\{\tilde{\nabla}_{E_{a}}\left(\mathrm{~d} \varphi\left(E_{a}\right)\right)-\mathrm{d} \varphi\left(\nabla_{E_{a}}^{g} E_{a}\right)\right\} \\
& =\sum_{a=1}^{q}\left\{\tilde{\nabla}_{E_{a}}\left(\mathrm{~d} \varphi\left(E_{a}\right)\right)-\mathrm{d} \varphi\left(\pi\left(\nabla_{E_{a}}^{g} E_{a}\right)\right)\right\}-\mathrm{d} \varphi\left(\left(\sum_{a=1}^{q} \nabla_{E_{a}}^{g} E_{a}\right)^{\perp}\right),
\end{aligned}
$$

the right hand side of (4.11) coincides with

$$
\int_{M}\left\langle\tau_{b}(\varphi)+\mathrm{d} \varphi\left(\left(\sum_{a=1}^{q} \nabla_{E_{a}}^{g} E_{a}\right)^{\perp}\right), \tau_{b}(\varphi)\right\rangle v_{g}
$$

(4.11) is equivalent to that

$$
\int_{M}\left\langle\mathrm{~d} \varphi\left(\kappa^{\#}\right), \tau_{b}(\varphi)\right\rangle v_{g}=\int_{M}\left\langle\tau_{b}(\varphi), \tau_{b}(\varphi)\right\rangle v_{g}+\int_{M}\left\langle\mathrm{~d} \varphi\left(\left(\sum_{a=1}^{q} \nabla_{E_{a}}^{g} E_{a}\right)^{\perp}\right), \tau_{b}(\varphi)\right) v_{g} .
$$

If $\varphi:(M, g) \rightarrow\left(M^{\prime}, g^{\prime}\right)$ is an isometric immersion, then it holds that $\left\langle\mathrm{d} \varphi_{x}\left(T_{x} M\right), \tau_{b}(\varphi)\right\rangle=0$, which implies that both the left hand side and the second term of the right hand side of (4.14) vanish, that is, $\int_{M}\left\langle\tau_{b}(\varphi), \tau_{b}(\varphi)\right\rangle v_{g}=0$. Therefore $\tau_{b}(\varphi) \equiv 0$.

We obtain Theorem 2.6.

\section{Principal $G$-Bundles}

In this section, we show the following theorem which is quite new and the more detail [34] will appear elsewhere.

Theorem 5.1 Let $\pi:(P, g) \rightarrow(M, h)$ be a principal G-bundle over a Riemannian manifold $(M, h)$ whose Ricci tensor is negative definite. Then, if $\pi:(P, g) \rightarrow(M, h)$ is biharmonic, then it is harmonic.

Let us consider a principal $G$-bundle $\pi:(P, g) \rightarrow(M, h)$ whose the total space $P$ is compact. Assume that the projection $\pi:(P, g) \rightarrow(M, h)$ is biharmonic, which is by definition, $J(\tau(\pi)) \equiv 0$, where $\tau(\pi)$ is the tension field of $\pi$ which is defined by

$$
\tau(\pi):=\sum_{i=1}^{p}\left\{\nabla_{e_{i}}^{h} \pi_{*} e_{i}-\pi_{*}\left(\nabla_{e_{i}}^{g} e_{i}\right)\right\},
$$

the Jacobi operator $J$ is defined by

$$
J V:=\bar{\Delta} V-\mathcal{R}(V) \quad\left(V \in \Gamma\left(\pi^{-1} T N\right)\right),
$$

$\bar{\Delta}$ is the rough Laplacian defined by

$$
\bar{\Delta} V:=-\sum_{i=1}^{p}\left\{\bar{\nabla}_{e_{i}}\left(\bar{\nabla}_{e_{i}} V\right)-\bar{\nabla}_{\nabla_{e_{i}}^{g} e_{i}} V\right\}
$$

and

$$
\mathcal{R}(V):=R^{h}\left(V, \pi_{*} e_{i}\right) \pi_{*} e_{i},
$$


where $\left\{e_{i}\right\}_{i=1}^{p}$ is a locally defined orthonormal frame field on $(P, g)$.

The tangent space $P_{u} \quad(u \in P)$ is canonically decomposed into the orthogonal direct sum of the vertical subspace $G_{u}=\left\{A_{u}^{*} \mid A \in \mathfrak{g}\right\}$ and the horizontal subspace $H_{u}$ : $P_{u}=G_{u} \oplus H_{u}$. Then, we have

$$
\begin{aligned}
\tau_{2}(\pi) & =\bar{\Delta} \tau(\pi)-\sum_{i=1}^{p} R^{h}\left(\tau(\pi), \pi_{*} e_{i}\right) \pi_{*} e_{i} \\
& =\bar{\Delta} \tau(\pi)-\sum_{i=1}^{m} R^{h}\left(\tau(\pi), \pi_{*} e_{i}\right) \pi_{*} e_{i}-\sum_{i=1}^{k} R^{h}\left(\tau(\pi), \pi_{*} A_{m+i}^{*}\right) \pi_{*} A_{m+i}^{*} \\
& =\bar{\Delta} \tau(\pi)-\sum_{i=1}^{m} R^{h}\left(\tau(\pi), \pi_{*} e_{i}\right) \pi_{*} e_{i}
\end{aligned}
$$

where $p=\operatorname{dim} P, m=\operatorname{dim} M, k=\operatorname{dim} G$, respectively. Then, we obtain

$$
\begin{aligned}
0 & =\int_{M}\langle J(\tau(\pi)), \tau(\pi)\rangle v_{g} \\
& =\int_{M}\left\langle\bar{\nabla}^{*} \bar{\nabla} \tau(\pi), \tau(\pi)\right\rangle v_{g}-\int_{M} \sum_{i=1}^{m}\left\langle R^{h}\left(\tau(\pi), \pi_{*} e_{i}\right) \pi_{*} e_{i}, \tau(\pi)\right\rangle v_{g} \\
& =\int_{M}\langle\bar{\nabla} \tau(\pi), \bar{\nabla} \tau(\pi)\rangle v_{g}-\int_{M} \sum_{i=1}^{m}\left\langle R^{h}\left(\tau(\pi), \pi_{*} e_{i}\right) \pi_{*} e_{i}, \tau(\pi)\right\rangle v_{g}
\end{aligned}
$$

Therefore, we obtain

$$
\begin{aligned}
\int_{M}\langle\bar{\nabla} \tau(\pi), \bar{\nabla} \tau(\pi)\rangle v_{g} & =\int_{M} \sum_{i=1}^{m}\left\langle R^{h}\left(\tau(\pi), \pi_{*} e_{i}\right) \pi_{*} e_{i}, \tau(\pi)\right\rangle v_{g} \\
& =\int_{M} \sum_{i=1}^{m} K^{h}\left(\tau(\pi), \pi_{*} e_{i}\right) \\
& =\int_{M} \operatorname{Ric}^{h}(\tau(\pi)) v_{g},
\end{aligned}
$$

where we denote by $K^{h}(u, v) \quad\left(u, v \in T_{x} M\right)$, the sectional curvature of $(M, h)$ through two plane of $T_{x} M$ given by $u, v \in T_{x} M$, and $\operatorname{Ric}(X)$ is the Ricci curvature of $(M, h)$ along $X \in T_{x} M$. The left hand side of (5.5) is non-negative, and then, the both hand sides of (5.5) must vanish if the Ricci curvature of $(M, h)$ is non-positive. Therefore, we obtain

$$
\left\{\begin{array}{l}
\bar{\nabla}_{X} \tau(\pi)=0 \quad(\forall X \in \mathfrak{X}(P)), \text { i.e., } \tau(\pi) \text { is parallel, and } \\
\operatorname{Ric}^{h}(\tau(\pi))=0 .
\end{array}\right.
$$

Let us consider a 1-form $\alpha$ on $M$ defined by

$$
\alpha(Y)(\pi(x)):=\left\langle\tau(\pi)(x), Y_{\pi(x)}\right\rangle,(Y \in \mathfrak{X}(M), x \in P) .
$$

Then, for every $Y, Z \in \mathfrak{X}(M)$, we have

$$
\begin{aligned}
\left(\nabla_{Z}^{h} \alpha\right)(Y) & =Z(\alpha(Y))-\alpha\left(\nabla_{Z}^{h} Y\right) \\
& =Z\langle\tau(\pi), Y\rangle-\left\langle\tau(\pi), \nabla_{Z}^{h} Y\right\rangle \\
& =\left\langle\bar{\nabla}_{Z} \tau(\pi), Y\right\rangle+\left\langle\tau(\pi), \nabla_{Z}^{h} Y\right\rangle-\left\langle\tau(\pi), \nabla_{Z}^{h} Y\right\rangle \\
& =0,
\end{aligned}
$$

which implies that $\alpha$ is parallel 1 -form on $(M, h)$. Since we assume that the Ricci 
tensor of $(M, h)$ is negative definite, $\alpha$ must vanish (so called Bochner's theorem, cf. [40], p. 55). Therefore, $\tau(\pi) \equiv 0$, i.e., the projection of the principal $G$-bundle $\pi:(P, g) \rightarrow(N, h)$ is harmonic. We obtain Theorem 5.1.

\section{Acknowledgements}

None.

\section{Funding}

Supported by the Grant-in-Aid for the Scientific Research, (C) No. 25400154, Japan Society for the Promotion of Science.

\section{References}

[1] Eells, J. and Lemaire, L. (1978) A Report on Harmonic Maps. Bulletin of London Mathematical Society, 10, 1-68. https://doi.org/10.1112/blms/10.1.1

[2] Eells, J. and Lemaire, L. (1983) Selected Topics in Harmonic Maps. CBMS Regional Conference Series in Mathematics, 50. https://doi.org/10.1090/cbms/050

[3] Eells, J. and Lemaire, L. (1988) Another Report on Harmonic Maps. Bulletin of London Mathematical Society, 20, 385-524. https://doi.org/10.1112/blms/20.5.385

[4] Jiang, G.Y. (2008) 2-Harmonic Maps and Their First and Second Variational Formulas. Note di Matematica, 28, 209-232. https://doi.org/10.1285/i15900932v28n1supplp209

[5] Chen, B.Y. (1991) Some Open Problems and Conjectures on Submanifolds of Finite Type: Recent Development. Tamkang Journal of Mathematics, 45, 87-108. https://doi.org/10.5556/j.tkjm.45.2014.1564

[6] Ou, Y.-L. and Tang, L. (2012) On the Generalized Chen's Conjecture on Biharmonic Submanifolds. Michigan Mathematical Journal, 61, 531-542. https://doi.org/10.1307/mmj/1347040257

[7] Akutagawa, K. and Maeta, S. (2013) Biharmonic Properly Immersed Submanifolds in Euclidean Spaces. Geometriae Dedicata, 164, 351-355.

https://doi.org/10.1007/s10711-012-9778-1

[8] Defever, F. (1998) Hypersurfaces of $\mathbb{E}^{4}$ with Harmonic Mean Curvature Vetor. Mathematische Nachrichten, 196, 61-69. https://doi.org/10.1002/mana.19981960104

[9] Hasanis, T. and Vlachos, T. (1995) Hypersurfaces in $\mathbb{E}^{4}$ with Harmonic Mean Curvature Vector Field. Mathematische Nachrichten, 172, 145-169. https://doi.org/10.1002/mana.19951720112

[10] Ichiyama, T., Inoguchi, J. and Urakawa, H. (2009) Bi-Harmonic Maps and bi-Yang-Mills Fields. Note di Matematica, 1, 233-275. https://doi.org/10.1285/i15900932v28n1supplp233

[11] Ichiyama, T., Inoguchi, J. and Urakawa, H. (2010) Classifications and Isolation Phenomena of Biharmonic Maps and Bi-Yang-Mills Fields. Note di Matematica, 30, 15-48.

[12] Inoguchi, J. (2004) Submanifolds with Harmonic Mean Curvature Vector Filed in Contact 3-Manifolds. Colloquium Mathematicum, 100, 163-179. https://doi.org/10.4064/cm100-2-2

[13] Ishihara, S. and Ishikawa, S. (1975) Notes on Relatively Harmonic Immersions. Hokkaido Mathematical Journal, 4, 234-246. https://doi.org/10.14492/hokmj/1381758762

[14] Nakauchi, N. and Urakawa, H. (2011) Biharmonic Hypersurfaces in a Riemannian Mani- 
fold with Non-Positive Ricci Curvature. Annals of Global Analysis and Geometry, 40, 125 131. https://doi.org/10.1007/s10455-011-9249-1

[15] Nakauchi, N. and Urakawa, H. (2013) Biharmonic Submanifolds in a Riemannian Manifold with Non-Positive Curvature. Results in Mathematics, 63, 467-474.

https://doi.org/10.1007/s00025-011-0209-7

[16] Nakauchi, N., Urakawa, H. and Gudmundsson, S. (2014) Biharmonic Maps into a Riemannian Manifold of Non-Positive Curvature. Geometriae Dedicata, 169, 263-272. https://doi.org/10.1007/s10711-013-9854-1

[17] Ohno, S., Sakai, T. and Urakawa, H. (2015) Biharmonic Homogeneous Hypersurfaces in Compact Symmetric Spaces. Differ. Differential Geometry and Its Applications, 43, 155 179. https://doi.org/10.1016/j.difgeo.2015.09.005

[18] Barletta, E., Dragomir, S. and Urakawa, H. (2001) Pseudoharmonic Maps from a Nondegenerate CR Manifold into a Riemannian Manifold. Indiana University Mathematics Journal, 50, 719-746. https://doi.org/10.1512/iumj.2001.50.1931

[19] Dragomir, S. and Montaldo, S. (2014) Subelliptic Biharmonic Maps. Journal of Geometric Analysis, 24, 223-245. https://doi.org/10.1007/s12220-012-9335-Z

[20] Urakawa, H. (2016) CR Rigidity of Pseudo Harmonic Maps and Pseudo Biharmonic Maps. Hokkaido Mathematical Journal.

[21] Chiang, Y.-J. and Wolak, R.A. (2008) Transversally Biharmonic Maps between Foliated Riemannian Manifolds. International Journal of Mathematics, 19, 981-996. https://doi.org/10.1142/S0129167X08004972

[22] Jung, S.D., Kim, B.H. and Pak, J.S. (2004) Lower Bounds for the Eigenvalues of the Basic Dirac Operator on a Riemannian Foliation. Journal of Geometry and Physics, 51, 166-182. https://doi.org/10.1016/j.geomphys.2003.10.004

[23] Alvarez Lopez, J.A. (1992) The Basic Component of the Mean Curvature of Riemannian Foliations. Ann. Annals of Global Analysis and Geometry, 10, 179-194. https://doi.org/10.1007/BF00130919

[24] Nishikawa, S., Tondeur, P.H. and Vanhecke, L. (1992) Spectral Geometry for Riemannian Foliations. Annals of Global Analysis and Geometry, 10, 291-304. https://doi.org/10.1007/BF00136871

[25] Park, J.H. (1990) The Laplace-Beltrami Operator and Riemannian Submersion with Minimal and Not Totally Geodesic Fibers. Bulletin of the Korean Mathematical Society, 27, 3947.

[26] Park, E. and Richardson, K. (1996) The Basic Laplacian of a Riemannian Foliation. American Journal of Mathematics, 118, 1249-1275. https://doi.org/10.1353/ajm.1996.0053

[27] Konderak, J.J. and Wolak, R. (2003) Transversally Harmonic Maps between Manifolds with Riemannian Foliations. Quarterly Journal of Mathematics, 54, 335-354. https://doi.org/10.1093/qmath/hag019

[28] Konderak, J.J. and Wolak, R. (2003) Some Remarks on Transversally Harmonic Maps. Glasgow Mathematical Journal, 50, 1-16.

[29] Jung, S.D. (2001) The First Eigenvalue of the Transversal Dirac Operator. Journal of Geometry and Physics, 39, 253-264. https://doi.org/10.1016/S0393-0440(01)00014-6

[30] Jung, S.D. (2007) Eigenvalue Estimates for the Basic Dirac Operator on a Riemannian Foliation Admitting a Basic Harmonic 1-Form. Journal of Geometry and Physics, 57, 12391246. https://doi.org/10.1016/j.geomphys.2006.04.008

[31] Jung, M.J. and Jung, S.D. (2012) On Transversally Harmonic Maps of Foliated Riemannian 
Manifolds. Journal of the Korean Mathematical Society, 49, 977-991.

[32] Jung, S.D. (2013) Variation Formulas for Transversally Harmonic and Bi-Harmonic Maps. Journal of Geometry and Physics, 70, 9-20.

[33] Ohno, S., Sakai, T. and Urakawa, H. (2016) Rigidity of Transversally Biharmonic Maps between Foliated Riemannian Manifolds. Hokkaido Mathematical Journal.

[34] Urakawa, H. (2016) Harmonic Maps and Biharmonic Maps on Principal G-Bundles.

[35] Dragomir, S. and Tomassini, G. (2006) Differential Geometry and Analysis on CR Manifolds. In: Chambert-Loir, A., et al., Eds., Progress in Mathematics, Vol. 246, Birkhäuser, Boston.

[36] Yorozu, S. and Tanemura, T. (1990) Green's Theorem on a Foliated Riemannian Manifold and Its Applications. Acta Mathematica Hungarica, 56, 239-245. https://doi.org/10.1007/BF01903838

[37] Tondeur, P.H. (1988) Foliations on Riemannian Manifolds. Springer, New York. https://doi.org/10.1007/978-1-4613-8780-0

[38] Tondeur, P.H. (1997) Geometry of Foliations. Birkhäuser, Basel.

[39] Gaffney, M.F. (1954) A Special Stokes' Theorem for Complete Riemannian Manifolds. Annals of Mathematics, 60, 140-145. https://doi.org/10.2307/1969703

[40] Kobayashi, S. (1972) Transformation Groups in Differential Geometry. Springer, Berlin. https://doi.org/10.1007/978-3-642-61981-6

Submit or recommend next manuscript to SCIRP and we will provide best service sfor you:

Accepting pre-submission inquiries through Email, Facebook, LinkedIn, Twitter, etc.

A wide selection of journals (inclusive of 9 subjects, more than 200 journals)

Providing 24-hour high-quality service

User-friendly online submission system

Fair and swift peer-review system

Efficient typesetting and proofreading procedure

Display of the result of downloads and visits, as well as the number of cited articles

Maximum dissemination of your research work

Submit your manuscript at: http://papersubmission.scirp.org/

Or contact jamp@scirp.org 